BULL. AUSTRAL. MATH. SOC.

VOL. 20 (1979), 407-410.

\title{
A note on rational approximation
}

\section{C.S. Davis}

It is shown that the inequality

$$
|e-(p / q)|<\frac{1}{2}\left((\log \log q) /\left(q^{2} \log q\right)\right)
$$

holds for an infinity of integers $p, q$ and that here the factor $\frac{2}{2}$ may not be replaced by a smaller number. Corresponding best possible inequalities are given for the numbers $e^{ \pm 2 / t}$, where $t$ is a positive integer.

In a recent paper (Davis [2]), the author gave the following result on approximation by rationals to numbers of the form $e^{ \pm 2 / t}$, where $t$ is a positive integer.

THEOREM. If $a= \pm 2 / t$, where $t \in \mathbb{N}$, and

$$
c=\left\{\begin{array}{lll}
1 / t, & t \text { even, } \\
1 /(4 t), & t \text { odd, }
\end{array}\right.
$$

then, for any $\varepsilon>0$, the inequality

$$
\left|e^{a}-(p / q)\right|<(c+\varepsilon)\left((\log \log q) /\left(q^{2} \log q\right)\right)
$$

has an infinity of solutions in integers $p, q$. Further, there exists a number $q^{\prime}$, depending only on $\varepsilon$ and $t$, such that

$$
\left|e^{a}-(p / q)\right|>(c-\varepsilon)\left((\log \log q) /\left(q^{2} \log q\right)\right)
$$

for all integers $p, q$ with $q \geq q^{\prime}$.

The second statement of the theorem shows that the constant $c$ in the

Received 18 April 1979. 
inequality (I) is 'best possible' in the sense that it can not be replaced by any smaller number. Nonetheless, the inequality (1) may be improved, in that $c+\varepsilon$ may be replaced by $c$, and it is the purpose of this note to establish this result, thus giving the

THEOREM. If $a= \pm 2 / t$, where $t \in \mathbb{N}$, and

$$
c= \begin{cases}1 / t, & t \text { even, } \\ 1 /(4 t), & t \text { odd, }\end{cases}
$$

then the inequality

$$
\left|e^{a}-(p / q)\right|<c\left((\log \log q) /\left(q^{2} \log q\right)\right)
$$

has an infinity of solutions in integers $p, q$. If $c$ be replaced by any smaller number, the inequality has only a finite number of integer solutions.

In the paper cited, details of the proof were given for the case $a=I$ (in which case $c=\frac{1}{2}$ ). The inequality (I) was established by explicitly constructing integers $P_{n}, Q_{n}$, for each $n \in \mathbb{N}$, such that

$$
\left|e-\left(p_{n} / Q_{n}\right)\right|=\left|J_{n}\right| / Q_{n}^{2}
$$

where $\left|J_{n}\right| \sim 1 / 2 n$ and $Q_{n} \sim V(2 / e)(4 n / e)^{n}$ as $n \rightarrow \infty$. The result (I) of the theorem follows, on taking $p=P_{n}, q=Q_{n}$, and observing that $n \sim\left(\log Q_{n}\right) /\left(\log \log Q_{n}\right)$. However, in the course of proving the second statement of the theorem it is shown that $P_{n} / Q_{n}$ is that convergent of the simple (or regular) continued fraction

$$
e=[2, \overline{1,2 n, I}]_{n=1}^{\infty}
$$

which arises by terminating that fraction immediately before the partial quotient $m$. Hence

$$
|e-(p / q)|<1 / 2 n q^{2}
$$

Now

$$
\begin{aligned}
\log q & =n \log n+O(n) \\
& =n \log n\{1+O(1 /(\log n))\}
\end{aligned}
$$


so

$$
\begin{aligned}
\log \log q & =\log n+\log \log n+o(1 /(\log n)) \\
& =(\log q) / n+\log \log n+o(1),
\end{aligned}
$$

and hence

$$
1 / n<(\log \log q) /(\log q)
$$

for all sufficiently large $n$. Thus

$$
|e-(p / q)|<\frac{1}{2}\left((\log \log q) /\left(q^{2} \log q\right)\right)
$$

for an infinity of $p, q$, as asserted.

We observe here, for later use, that (3) may be replaced by

$$
1 /(n-m)<(\log \log q) /(\log q) \text {, }
$$

for any bounded $m$, since, by (2),

$$
\log q=(n-m) \log n+o(n) \text {. }
$$

In order to complete the proof to cover other values of $a$, we quote relevant results from Davis [1]. We denote by $a_{n}, p_{n} / q_{n}(n=0,1, \ldots)$ respectively the partial quotients and convergents of the continued fractions in question. Further, we observe that our $Q_{n}$ is the $B_{n, n}$ of the paper just cited and that hence

$$
Q_{n} \sim(4 n / a e)^{n} \vee\left(2 e^{-a}\right)
$$

Thus if we take $q=Q_{n}$ (or $\frac{1}{2} Q_{n}$, if appropriate), the inequality (4) still holds.

For $a=2 / t$ with $t$ even, say $t=2 k$, and $k>1$, we have $a_{3 n-2}=(2 n-1) k-1$ and take $q=q_{3 n-3}=Q_{n}$. Noting that

$$
a_{3 n-2}=2 n k-(k+1)>2 n k-2 k=t(n-1) \text {, }
$$

we have

$$
\left|e^{a}-(p / q)\right|<1 /\left(t(n-1) q^{2}\right)
$$

and the result follows, on using (4).

The case $a=2 / t$ with $t$ odd is a little more complicated in detail 
and, for simplicity, we write $3 n+1=N$. Then

$$
\begin{aligned}
& \text { (i) if } t=1, a_{5 n}=6(2 n+1)=4 N+2, \\
& q=q_{5 n-1}=\frac{1}{2} Q_{3 n+1}=\frac{3}{2} Q_{N} ; \\
& \text { (ii) if } t>1, a_{5 n+2}=6 t(2 n+1)=4 t N+2 t, \\
& q=q_{5 n+1}=\frac{1}{2} Q_{3 n+1}=\frac{1}{2} Q_{N} .
\end{aligned}
$$

The result in this case follows as before.

Finally, the case of $e^{-a}$ with $\alpha>0$ is essentially the same, since here we simply take $q=p_{K-1}$ instead of $q_{K}$ (the notation referring to the continued fraction for the corresponding $e^{a}$ ).

\section{References}

[1] C.S. Davis, "On some simple continued fractions connected with $e$ ", J. London Math. Soc. 20 (1945), 194-198.

[2] C.S. Davis, "Rational approximations to e ", J. Austral. Math. Soc. Ser. A 25 (1978), 497-502.

Department of Mathematics, University of Queensland,

St Lucia,

Queensland. 